\title{
Experimental measurements of rf breakdowns and deflecting gradients in mm-wave metallic accelerating structures
}

\author{
Massimo Dal Forno, ${ }^{1,}$ Valery Dolgashev, ${ }^{1}$ Gordon Bowden, ${ }^{1}$ Christine Clarke, ${ }^{1}$ \\ Mark Hogan, ${ }^{1}$ Doug McCormick, ${ }^{1}$ Alexander Novokhatski, ${ }^{1}$ Bruno Spataro, ${ }^{2}$ \\ Stephen Weathersby, ${ }^{1}$ and Sami G. Tantawi ${ }^{1}$ \\ ${ }^{1}$ SLAC National Accelerator Laboratory, Menlo Park, California 94025, USA \\ ${ }^{2}$ INFN/LNF, Frascati (Roma), 00044, Italy \\ (Received 6 August 2015; published 3 May 2016)
}

\begin{abstract}
We present an experimental study of a high gradient metallic accelerating structure at sub- $\mathrm{THz}$ frequencies, where we investigated the physics of rf breakdowns. Wakefields in the structure were excited by an ultrarelativistic electron beam. We present the first quantitative measurements of gradients and metal vacuum rf breakdowns in sub- $\mathrm{THz}$ accelerating cavities. When the beam travels off axis, a deflecting field is induced in addition to the longitudinal field. We measured the deflecting forces by observing the displacement and changes in the shape of the electron bunch. This behavior can be exploited for subfemtosecond beam diagnostics.
\end{abstract}

DOI: 10.1103/PhysRevAccelBeams.19.051302

Accelerating gradient is one of the crucial parameters affecting the design, construction and cost of the next generation of linear accelerators. To reach high gradient acceleration above the state of the art of $100 \mathrm{MV} / \mathrm{m}$, several problems such as vacuum rf breakdowns must be overcome [1,2]. During the development of the Next Linear Collider/Global Linear Collider the statistical nature of rf breakdown became apparent [2-4]. It was found that when accelerating structures are exposed to constant rf power and pulse shape, the number of rf breakdowns per pulse is nearly constant. The breakdown rate depends on pulse heating [5] and other factors, such as the peak magnetic field [6], the peak electric field, and the peak Poynting vector [7].

Presently X-band accelerating structures are the most studied in terms of rf breakdowns [2,8,9]. Currently, research in accelerating structures is moving towards higher rf frequencies because of the expected higher gradients. However, data on breakdown statistics above $40 \mathrm{GHz}$ is not available [10-12].

In the 1990s, mm-wave metal and dielectric accelerating structures were considered for linear colliders [1,13]. Hill et al. $[14,15]$ tested both a single metal mm-wave cavity and a dielectric accelerator structure excited by an electron beam. Henke and Bruns [16-18] and Chou and Kroll at SLAC [19] designed a mm-wave muffin-tin planar accelerator structure. Another approach to reach higher

"dalforno@slac.stanford.edu

Published by the American Physical Society under the terms of the Creative Commons Attribution 3.0 License. Further distribution of this work must maintain attribution to the author $(s)$ and the published article's title, journal citation, and DOI. accelerating gradients at higher frequencies involves dielectric wakefield accelerators [20-22]. At Argonne these were tested as well as dielectric wakefield-excited structures [23-26]. To our knowledge, in those experiments there were no systematic studies of rf breakdown probability.

In this paper we present a quantitative measurement of $\mathrm{rf}$ breakdowns and gradients in a mm-wave copper traveling wave structure. The major goal of our study is to determine the breakdown properties and specifically how the $\mathrm{rf}$ breakdown rate changes with if parameters at these high frequencies. Moreover, when the electron beam travels off axis, a deflecting field is induced in addition to the longitudinal field. We measured the deflecting forces by observing the displacement and changes in the shape of the electron bunch. This behavior can be exploited for subfemtosecond beam diagnostics [27].

A set of $120 \mathrm{GHz}$ traveling wave constant impedance accelerating structures was designed, built and tested [28]. The structures were excited by the ultrarelativistic electron beam generated by the facility for advanced accelerator experimental tests (FACET) [29]. The novelty of this paper is the measurement of the breakdown rate and the measurement of the electron beam transverse deflection. The studied structure is open, made of two separate metal blocks. It is $10 \mathrm{~cm}$ long and composed of 125 coupled cavities. Cavities are milled into the flat face of each block. The two halves are placed together, with a gap between, forming an open accelerating structure [see Fig. 1(a)]. Figure 1(b) shows one half of the structure, and Fig. 1(c) shows the geometry of one quarter of the vacuum volume of a regular cell. The $x$ direction is horizontal, while the $y$ direction is vertical. The fundamental mode is synchronous with the speed of light at a phase advance per period close to $2 \pi / 3$. Radio-frequency parameters for the fundamental 


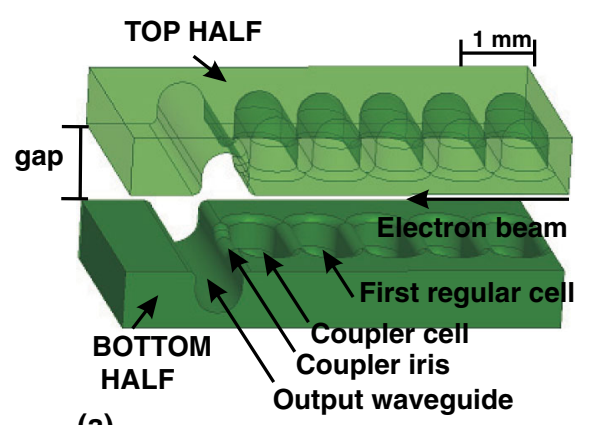

(a)

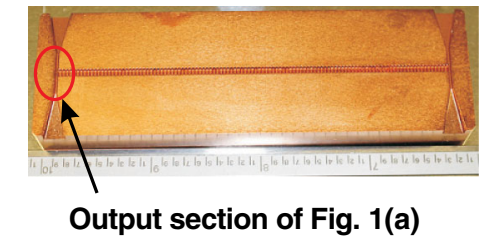

(b)

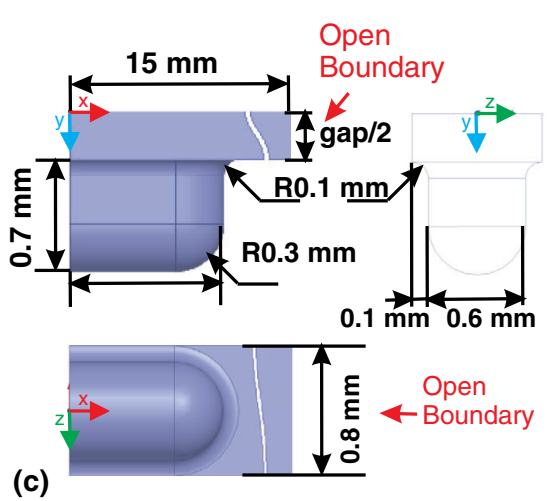

FIG. 1. Solid model of the output section of the mm-wave traveling wave accelerating structure, including output coupler and waveguides (a), photo of the copper half structure (b), geometry of one quarter of the vacuum part of the regular cell (c).

accelerating mode are listed in Table I. They were calculated for a beam on the central axis, using the method described in [28]. The peak electric and magnetic fields are calculated on the surface. Their distribution, for the fundamental mode, is shown in Fig. 2. To calculate the pulse length we consider fields near the output (downstream) waveguide. Only the fundamental mode is considered, which has highest loss factor and lower attenuation at frequencies around $100 \mathrm{GHz}$. As the fundamental mode propagates towards the output waveguide its energy decays due to losses in the metal. Therefore the field at the output waveguide is decaying with time constant depending on $Q_{0}$ and group velocity $v_{g}$, with total pulse length equal to $L / v_{g} \cdot\left(1-v_{g} / c\right)$. During the experiment we varied the rf parameters of the structure by remotely changing the gap. With larger gap the pulse length is determined by the group velocity and structure length, and with smaller gap and thus higher attenuation the pulse length is equal to the decay time [28].

Accelerating gradient was varied by horizontally scanning the beam position with respect to the structure center axis. To reliably detect breakdowns we used a diagnostic tool which we call "arc-detector" [28]. The two metal halves were electrically insulated from ground and each other. Radio-frequency breakdowns generate electron and ion currents that induce a voltage between the two halves, which were measured with an oscilloscope.

When the electron beam trajectory moves horizontally off axis it excites deflecting fields, however when the beam trajectory moves far beyond the corrugations, the deflection disappears. We simulated wakefields excited by a Gaussian bunch with $\sigma_{z}=50 \mu \mathrm{m}$ and charge $3.2 \mathrm{nC}$ using CST Particle Studio [30]. The integrated accelerating and deflecting voltages in the $10 \mathrm{~cm}$ long structure are shown in Fig. 3. These results are in good agreement with other wakefield calculations made with a method developed by Novokhatski [31-34]. When the beam trajectory is close to the central axis there is no deflecting voltage. The deflection appears only as two spikes which are far from the structure center and close to the edge of the cavities.

The structure was installed in the vacuum chamber located in the experimental FACET section, shown in Fig. 4. A camera located after the vertically bending magnet records the screen image of the bunch at each pulse. The vertical screen coordinate corresponds to the beam energy. The beam optics between the test structure and the camera converts the horizontal kick angle

TABLE I. Radio-frequency parameters of the fundamental mode in the traveling wave copper accelerating structure. Radio-frequency fields and power are normalized to $1 \mathrm{nC}$ bunch charge. The beam is on the central axis. The peak electric and magnetic fields are calculated on the surface, " $1 m$." means for the fundamental mode only, while "all $m$." means the sum of all modes.

\begin{tabular}{|c|c|c|c|c|c|c|c|c|c|c|c|}
\hline Gap & Frequency & $\begin{array}{l}\text { Shunt } \\
\text { impedance }\end{array}$ & $\begin{array}{l}\text { Loss } \\
\text { factor }\end{array}$ & $\begin{array}{l}\text { Group } \\
\text { velocity }\end{array}$ & $\begin{array}{l}\text { Pulse } \\
\text { length }\end{array}$ & $\begin{array}{l}\text { Output } \\
\text { power }\end{array}$ & $\begin{array}{l}\text { Accelerating } \\
\text { gradient }\end{array}$ & $\begin{array}{l}E_{\text {peak }} \\
\text { surface }\end{array}$ & $\begin{array}{l}H_{\text {peak }} \\
\text { surface }\end{array}$ & $\begin{array}{l}\text { Breakdown rate } \\
\text { (@ } E_{\max , \text { allm } m} \text {.) }\end{array}$ & $\begin{array}{l}\text { No. of } \\
\text { pulses }\end{array}$ \\
\hline & $f$ & $R_{s}$ & $\kappa$ & $v_{g} / c$ & $\tau_{p}$ & $P$ & $E_{\mathrm{acc}}$ & $E_{\max , 1 m}$ & $H_{\max , 1 m}$ & BDR & \\
\hline $\mathrm{mm}$ & $\mathrm{GHz}$ & $\mathrm{M} \Omega / \mathrm{m}$ & $\mathrm{MV} / \mathrm{nC} / \mathrm{m}$ & $\%$ & ns & MW & $\mathrm{MV} / \mathrm{m}$ & $\mathrm{GV} / \mathrm{m}$ & $\mathrm{MA} / \mathrm{m}$ & {$[1 /($ pulse $\mathrm{m})]$} & \\
\hline 0.2 & 140.28 & 449 & 47.45 & 0.21 & 2.34 & 0.03 & 95 & 0.2 & 0.4 & & \\
\hline 0.3 & 136.27 & 398 & 41.60 & 0.85 & 2.36 & 0.105 & 83 & 0.19 & 0.356 & & \\
\hline 0.5 & 130.30 & 298 & 31.15 & 3.54 & 2.37 & 0.34 & 62.5 & 0.174 & 0.347 & & \\
\hline 0.7 & 126.01 & 222 & 23.50 & 7.65 & 4.03 & 0.58 & 47 & 0.16 & 0.34 & & \\
\hline 0.9 & 122.66 & 166 & 17.87 & 12.42 & 2.35 & 0.75 & 36 & 0.15 & 0.3 & 0.66 (@0.57 GV/m) & 3200 \\
\hline 1.1 & 119.93 & 128 & 13.89 & 17.48 & 1.57 & 0.88 & 28 & 0.12 & 0.23 & $0.38(@ 0.47$ GV/m) & 500 \\
\hline 1.3 & 117.59 & 99 & 10.97 & 22.44 & 1.15 & 0.94 & 22 & 0.11 & 0.2 & & \\
\hline
\end{tabular}




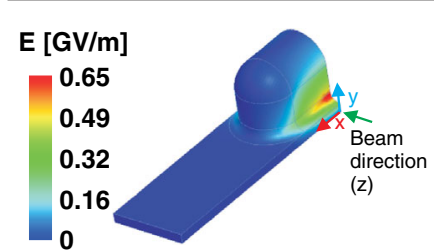

(a)

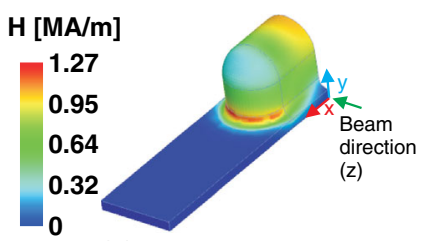

(b)

FIG. 2. Plot of the electric field (a) and magnetic field (b) of the fundamental mode over one quarter of the period of the regular cell (gap $=0.2 \mathrm{~mm}$ ), at synchronous frequency. These fields are calculated for a bunch charge of $3.2 \mathrm{nC}$ and $50 \mu \mathrm{m}$ long in an infinite traveling wave structure.

$\theta_{x}$, generated by the structure, to a horizontal beam displacement $\Delta x$, given by

$$
\Delta x=R_{12} \cdot \theta_{x}=R_{12} \cdot \frac{e V_{x}}{E},
$$

where $e$ is the electron charge, $R_{12}(=14 \mathrm{~m})$ is the optics coefficient that converts a beam horizontal angle into a beam horizontal displacement, given by the deflecting voltage $V_{x}$ and the beam energy $E$. By measuring $\Delta x$ on the diagnostic screen, the deflecting voltage is determined.

The FACET beam had an energy $E=20.35 \mathrm{GeV}$, a charge of $3.2 \mathrm{nC}$ and an rms bunch length of about $50 \mu \mathrm{m}$. During the experiment the projected emittances near the experimental chamber were approximately $\epsilon_{x}=$ $100 \mathrm{~mm}$ mrad and $\epsilon_{y}=10 \mathrm{~mm}$ mrad and transverse sizes were measured with: $\sigma_{x}=\sigma_{y}=35 \mu \mathrm{m}$.

To vary the structure properties and gradients, we first remotely set the gap between the two blocks and then horizontally moved the structure position with respect to the beam, changing the gradient. In the experiment, there were four gap settings of $1.3,1.1,0.9$, and $0.7 \mathrm{~mm}$, for which the rf parameters are listed in Table I.

During a horizontal scan the beam is decelerated and horizontally deflected. We were not able to detect any vertical beam displacement on the dispersive screen, which would be caused by beam deceleration because, with the FACET $20 \mathrm{GeV}$ beam, the resolution of the spectrometer did not allow its direct measurement. We could clearly observe horizontal deflection of the beam centroid, from which the deflecting voltage was calculated using $R_{12}$.

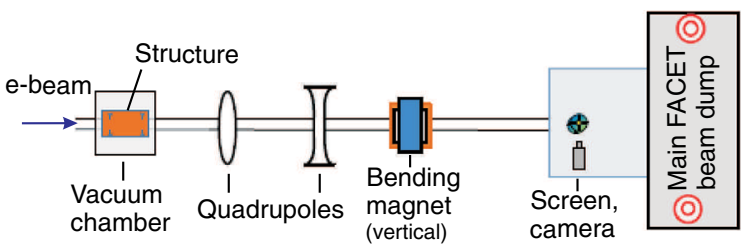

FIG. 4. Schematic of the experimental FACET section.

Figure 5 shows this analysis of a horizontal scan. Vertical error bars correspond to standard deviation of the centroid positions caused by transverse position jitter of the FACET bunch, pulse to pulse variation of the bunch shape and $\mathrm{rf}$ breakdowns. We believe that good correspondence between the simulated and measured deflecting voltages confirms our simulations of the accelerating voltage.

Wakefields cannot deflect the head of the bunch. The deflecting field is mostly seen by the tail. In an attempt to determine the head-tail configuration of the bunch we analyzed each screen image [see Fig. 6(a)], slicing it at constant vertical values. We analyzed each horizontal slice and we found that the data is well fitted by a double Gaussian. Figure 6 shows a typical image (a), with an example of the reconstruction of a horizontal slice (b). We analyzed each image, obtaining the centroid positions of the two Gaussians for each horizontal slice. We selected three plots, where the tail deflection was expected to be the largest to the left and right, while no deflection is expected when the beam is far from the corrugations. When they were superimposed, we found that one set of Gaussian centers (represented by the dots) moves relative to itself between the three images, while the other set did not. This analysis is shown in Fig. 7. The part of the bunch that did not move is the head, while the other is the tail. The analysis of vertical slice of the image can give information about the bunch's longitudinal phase space $[27,35]$.

With the known gradient and data from the arc detector, two types of breakdown statistics were measured. In the first we put the beam on the central axis and exposed the structure to a large number of pulses. The results are summarized in Table I.

The second experiment was done during a horizontal scan where the scan starts clear of the corrugations. During

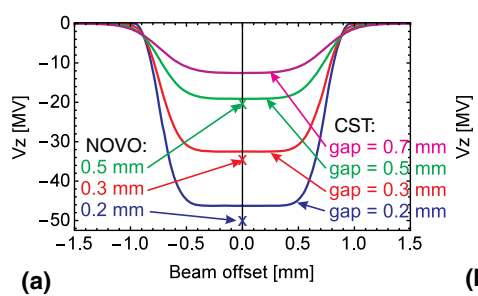

(a)

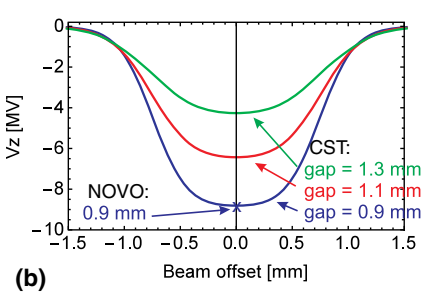

(b)

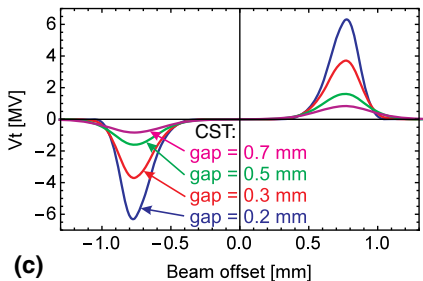

(c)

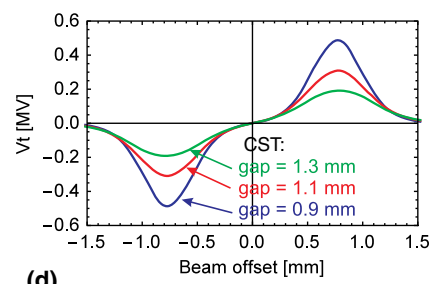

(d)

FIG. 3. Deceleration voltage [(a) and (b)] and transverse voltage [(c) and (d)] as a function of the horizontal beam-structure displacement, for different gaps. The fields are generated by a $3.2 \mathrm{nC}$ bunch with $\sigma_{z}=50 \mu \mathrm{m}$ in the $10 \mathrm{~cm}$ long structure. The solid lines are calculated with CST, the "X" points with the code NOVO. 


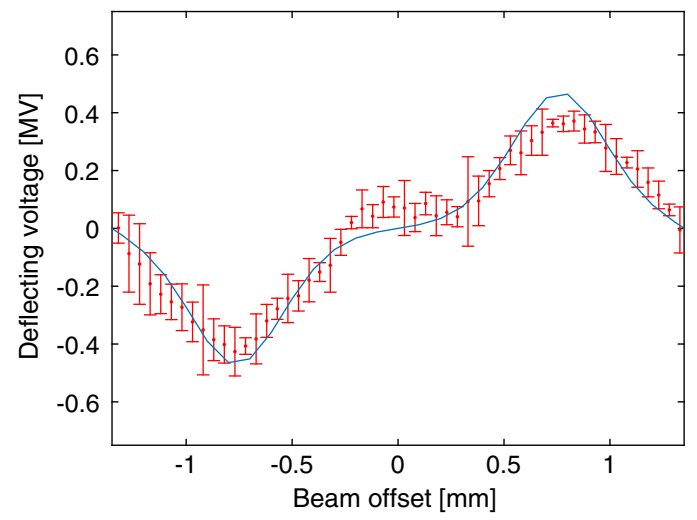

FIG. 5. Measurement of beam deflection in a horizontal scan with gap $=0.9 \mathrm{~mm}$. The blue line is the simulation.

the scan the gradient first increased as the beam approaches the center axis and then decreased (see Figs. 3 and 5). At each step we recorded the arc-detector data and then calculated the corresponding breakdown rate. The result of this measurement is shown in Fig. 8. The blue line is a linear fit of the first part of the scan where the gradient increased. The red line is the second part where the gradient decreased. Since the second part of the scan shows lower breakdown rates, we hypothesize it is due to rf conditioning, where the breakdown rate decreases after rf exposure.

Our tests were done in a large vacuum chamber, as a consequence, the vacuum pressure was $7 \times 10^{-7}$ Torr. We conjecture that $\mathrm{rf}$ conditioning and a lower vacuum pressure could reduce the measured breakdown rate.

Deflecting voltage was measured by observing the displacement of the electron beam on a diagnostic screen. This allowed us to confirm the simulated values of the deflecting and accelerating gradients. In our measurements we clearly see the difference between the deflection on the head and tail of the bunch. The deflecting wakefields create a chirp of deflecting forces over the length of the bunch,

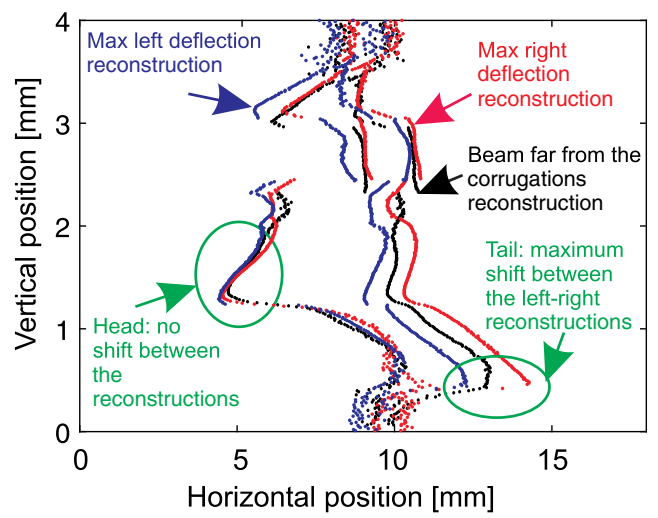

FIG. 7. Horizontal deflection of the bunch tail indicated by the horizontal shift of the centroids of double Gaussian fit. The blue dots are for the maximum left deflection, the red dots for the maximum right deflection and the black dots are for the undeflected beam, far from the corrugations. The bunch tail is clearly observed.

which could be used for bunch diagnostics at sub-fs resolution $[27,35]$.

By knowing the accelerating gradient of the structure, breakdown probability at rf frequencies near $120 \mathrm{GHz}$ was measured. At gap $=0.9 \mathrm{~mm}$, with a peak surface electric field of $0.57 \mathrm{GV} / \mathrm{m}$ (considering all modes), the breakdown rate is $0.66 /$ (pulse $\mathrm{m}$ ) for a pulse length of $2.35 \mathrm{~ns}$.

This experiment is the first in this parameter space where the rf breakdowns were measured. We plan to continuously study the experiments at different vacuum pressures and extend the conditioning time to improve our understanding of physics of $\mathrm{rf}$ breakdown at these high frequencies. As these studies are difficult to perform at FACET, we plan experiments with gyrotron driven accelerating structures where we will have flexibility to change vacuum pressure and conditioning procedure. The breakdown rate is expected to improve with a better vacuum and more conditioning time.
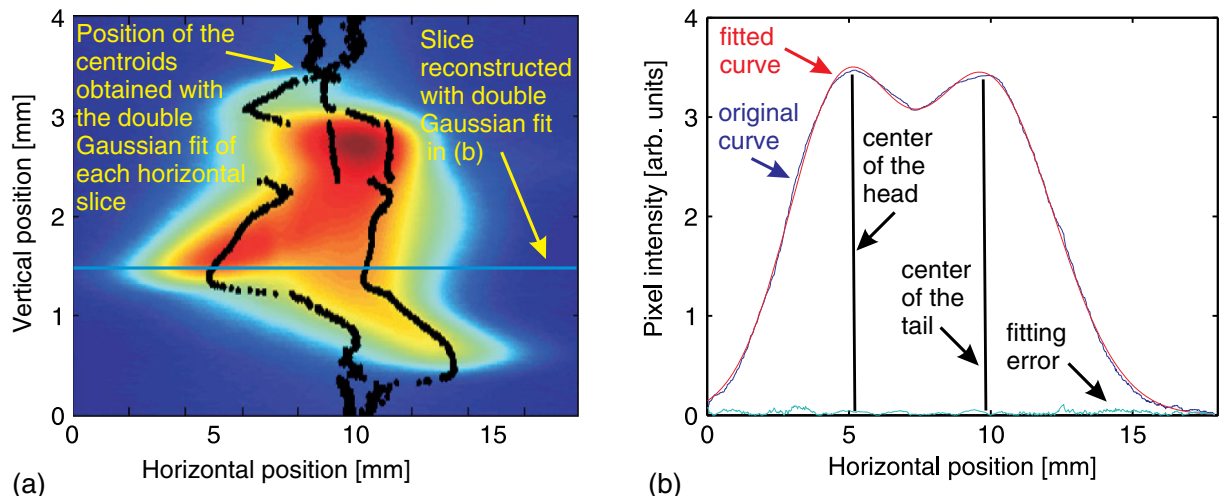

FIG. 6. Image of the beam recorded by the camera. The vertical axis is proportional to the energy, the zero is highest energy. The horizontal axis is the displacement. The dark points on the picture are the centroids obtained with the double Gaussian fit of each horizontal slice (a). Example of double Gaussian fit reconstruction of the horizontal slice, showing the two Gaussian centroids (b). 


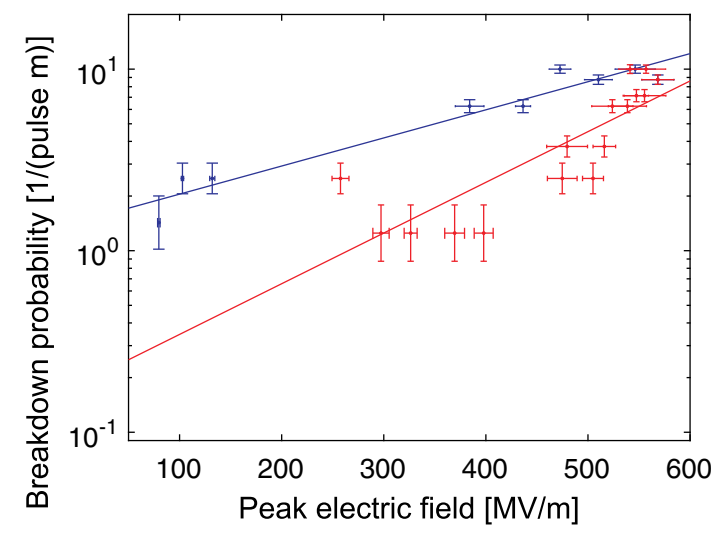

FIG. 8. Horizontal breakdown probability versus peak surface electric field obtained in a horizontal scan, $0.9 \mathrm{~mm}$ gap. The blue line is for increasing gradient and the red is for decreasing gradient. The electric peak field is calculated considering all modes.

The breakdown rates are relatively high for the field levels and pulse lengths as compared to values extrapolated from $\mathrm{x}$-band experiments $[2,10]$. X-band structures were conditioned by more than $10^{8} \mathrm{rf}$ pulses, typically without beam and vacuum pressures below $10^{-8}$ Torr. In this experiment the rf field is excited by the FACET beam so the number of pulses was limited to $<10^{6}$ and the vacuum level is $7 \times 10^{-7}$ Torr. The beam halo is intercepted by the structure and a few times, the whole beam was dumped into the structure due to linac faults. All of the above could contribute to a relatively high breakdown rate. These experiments are the first yielding quantitative measurements of rf breakdown rate in this parameter space. It will take many more experiments to understand the physics of it.

In conclusion this paper reports experimental measurements of deflecting forces and rf breakdown statistics in a copper traveling wave high gradient accelerating structure at sub-THz frequencies. This structure can be used as either a wakefield-driven or an rf powered accelerating structure. For the latter one, a gyroklystron would be a practical rf drive source.

This work was supported by the U.S. DOE under Contract No. DE-AC02-76SF00515. We thank the experiment crew: Brendan O'Shea, Spencer Gessner, Oliver Williams, Chris Pearson, Erik Adli, Carl Lindstrom, Michael Litos. We thank Vitaly Yakimenko and the FACET team for their excellent support.

[1] D. H. Whittum, Report No. SLAC-PUB-7809, 1998.

[2] V. A. Dolgashev, Progress on high-gradient structures, AIP Conf. Proc. 1507, 76 (2012).

[3] S. Doebert et al., High gradient performance of NLC/GLC $\mathrm{X}$-band accelerating structures, in Proceedings of the 21st
Particle Accelerator Conference, Knoxville, TN, 2005 (IEEE, Piscataway, NJ, 2005), pp. 372-374.

[4] C. Adolphsen, Normal conducting rf structure test facilities and results, in Proceedings of the 2003 Particle Accelerator Conference, Portland, OR (IEEE, New York, 2003), pp. 668-672.

[5] V. A. Dolgashev, High magnetic fields in couplers of $\mathrm{X}$-band accelerating structures, in Proceedings of the 2003 Particle Accelerator Conference, Portland, OR, pp. 1267-1269.

[6] V.A. Dolgashev and S. G. Tantawi, RF breakdown in $\mathrm{X}$-band waveguides, in Proceedings of the 8th European Particle Accelerator Conference, Paris, 2002 (EPS-IGA and CERN, Geneva, 2002), pp. 2139-2141.

[7] A. Grudiev, S. Calatroni, and W. Wuensch, New local field quantity describing the high gradient limit of accelerating structures, Phys. Rev. ST Accel. Beams 12, 102001 (2009).

[8] V. Dolgashev, S. Tantawi, Y. Higashi, and B. Spataro, Geometric dependence of radio-frequency breakdown in normal conducting accelerating structures, Appl. Phys. Lett. 97, 171501 (2010).

[9] F. Wang, C. Adolphsen, and C. Nantista, Performance limiting effects in X-band accelerators, Phys. Rev. ST Accel. Beams 14, 010401 (2011).

[10] H. H. Braun, S. Döbert, I. Wilson, and W. Wuensch, Frequency and Temperature Dependence of Electrical Breakdown at 21, 30, and $39 \mathrm{GHz}$, Phys. Rev. Lett. 90, 224801 (2003).

[11] D. Yu, H. Henke, H. H. Braun, S. Dobert, and W. Wuensch, High power test of a $30-\mathrm{GHz}$ planar accelerator, in Proceedings of the Particle Accelerator Conference, Chicago, IL, 2001 (IEEE, New York, 2001), Vol. 5, pp. 3858-3860.

[12] W.W. H. Braun and M. Valentini, CERN, Report No. CERN-CLIC-NOTE-413, 1999.

[13] D. Whittum, H. Henke, and P. Chou, High-gradient cavity beat-wave accelerator at W-band, in Proceedings of the Particle Accelerator Conference, Vancouver, BC, Canada, 1997 (IEEE, New York, 1997), Vol. 1, pp. 542-544.

[14] M. E. Hill, C. Adolphsen, W. Baumgartner, R. S. Callin, X.E. Lin, M. Seidel, T. Slaton, and D. H. Whittum, High-Gradient Millimeter-Wave Accelerator on a Planar Dielectric Substrate, Phys. Rev. Lett. 87, 094801 (2001).

[15] M. Hill, W. Fowkes, X. Lin, and D. Whittum, Beam-cavity interaction circuit at W-band, IEEE Trans. Microwave Theory Tech. 49, 998 (2001).

[16] H. Henke, Planar structures for electron acceleration, in Proceedings of the Particle Accelerator Conference, Dallas, TX, 1995 (IEEE, New York, 1995), Vol. 3, pp. 1750-1752.

[17] W. Bruns, Design of input couplers and endcells for side coupled muffin-tin structures, in Proceedings of the Particle Accelerator Conference, Dallas, TX, 1995 (Ref. [16]), Vol. 2, pp. 1088-1089.

[18] W. Bruns, Error sensitivity study for side coupled muffin tin structures using a finite difference program, in Proceedings of the Particle Accelerator Conference, Dallas, TX, 1995 (Ref. [16]), Vol. 2, pp. 1085-1087.

[19] SLAC W-band program, http://www.slac.stanford.edu/grp/ arb/tn/histpap/wband/. 
[20] G. Andonian, D. Stratakis, M. Babzien, S. Barber, M. Fedurin, E. Hemsing, K. Kusche, P. Muggli, B. O'Shea, X. Wei, O. Williams, V. Yakimenko, and J. B. Rosenzweig, Dielectric Wakefield Acceleration of a Relativistic Electron Beam in a Slab-Symmetric Dielectric Lined Waveguide, Phys. Rev. Lett. 108, 244801 (2012).

[21] M. C. Thompson, H. Badakov, A. M. Cook, J. B. Rosenzweig, R. Tikhoplav, G. Travish, I. Blumenfeld, M. J. Hogan, R. Ischebeck, N. Kirby, R. Siemann, D. Walz, P. Muggli, A. Scott, and R. B. Yoder, Breakdown Limits on Gigavolt-per-Meter Electron-Beam-Driven Wakefields in Dielectric Structures, Phys. Rev. Lett. 100, 214801 (2008).

[22] J. B. Rosenzweig, G. Andonian, P. Muggli, P. Niknejadi, G. Travish, O. Williams, K. Xuan, and V. Yakimenko, High frequency, high gradient dielectric wakefield acceleration experiments at SLAC and BNL, AIP Conf. Proc. 1299, 364 (2010).

[23] D. Wang, S. Antipov, C. Jing, J. G. Power, M. Conde, E. Wisniewski, W. Liu, J. Qiu, G. Ha, V. Dolgashev, C. Tang, and W. Gai, Interaction of an Ultrarelativistic Electron Bunch Train with a W-Band Accelerating Structure: High Power and High Gradient, Phys. Rev. Lett. 116, 054801 (2016).

[24] W. Gai, P. Schoessow, B. Cole, R. Konecny, J. Norem, J. Rosenzweig, and J. Simpson, Experimental Demonstration of Wake-Field Effects in Dielectric Structures, Phys. Rev. Lett. 61, 2756 (1988).

[25] M. Conde, S. Antipov, W. Gai, C. Jing, R. Konecny, W. Liu, J. Power, H. Wang, and Z. Yusof, The Argonne wakefield accelerator facility: Status and recent activities, in Proceedings of the 21st Particle Accelerator Conference, Knoxville, TN, 2005 (Ref. [3]), pp. 1485-1487.

[26] M. Conde, Survey of advanced dielectric wakefield accelerators, in Proceedings of the 22nd Particle Accelerator Conference, PAC-2007, Albuquerque, NM (IEEE, New York, 2007), pp. 1899-1903.

[27] S. Bettoni, P. Craievich, A. A. Lutman, and M. Pedrozzi, Temporal profile measurements of relativistic electron bunch based on wakefield generation, Phys. Rev. Accel. Beams 19, 021304 (2016).

[28] M. Dal Forno, V. Dolgashev, G. Bowden, C. Clarke, M. Hogan, D. McCormick, A. Novokhatski, B. Spataro, S. Weathersby, and S. G. Tantawi, rf breakdown tests of mm-wave metallic accelerating structures, Phys. Rev. Accel. Beams 19, 011301 (2016).

[29] Facility for advanced accelerator experimental tests (FACET), http://facet.slac.stanford.edu/.

[30] CST, Darmstadt, Germany, http://www.cst.de/.

[31] A. Novokhatsky, SLAC, Report No. SLAC-PUB-11556.

[32] A. Novokhatski, Field dynamics of coherent synchrotron radiation using a direct numerical solution of Maxwell's equations, Phys. Rev. ST Accel. Beams 14, 060707 (2011).

[33] A. Novokhatsky, SLAC, Report No. SLAC-PUB-15258, 2012.

[34] V. E. Balakin, I. A. Koop, A. V. Novokhatski, and V. P. Smirnov, SLAC, Report No. SLAC-TRANS-0188, 1978.

[35] V. Dolgashev, Attosecond diagnostics of multi-GeV electron beams using W-band deflectors, in Proceedings of FEIS-2: Femtosecond electron imaging and spectroscopy, East Lansing, Michigan, 2015, http://www.feis-2.org. 